\section{Kidney \\ Blood Pressure Research}

Kidney Blood Press Res 2017;42:1119-1127

DOI: $10.1159 / 000485779$

Published onlıne: December 07, 2017

Accepted: November 28, 2017

This article is licensed under the Creative Commons Attribution-NonCommercial-NoDerivatives 4.0 International License (CC BY-NC-ND) (http://www.karger.com/Services/OpenAccessLicense). Usage and distribution for commercial purposes as well as any distribution of modified material requires written permission.

\title{
Comparison of Muscle Mass Indices Using Computed Tomography or Dual X-Ray Absorptiometry for Predicting Physical Performance in Hemodialysis Patients
}

\author{
Seok Hui Kang ${ }^{a}$ Hyun Seok Lee ${ }^{b}$ Sukyung Lee Ji-Hyung Cho $^{d}$ Jun Chul Kim ${ }^{d}$ \\ aDivision of Nephrology, Department of Internal Medicine, Yeungnam University Hospital, Daegu, \\ 'Department of Radiology, CHA Gumi Medical Center, CHA University, Gumi-si, Gyeongsangbuk-do, \\ 'Division of Nephrology, Department of Internal Medicine, Semyung Christianity Hospital, Pohang-si, \\ Gyeongsangbuk-do, dDivision of Nephrology, Department of Internal Medicine, CHA Gumi Medical \\ Center, CHA University, Gumi-si, Gyeongsangbuk-do, Republic of Korea
}

\section{Key Words}

Hemodialysis - Thigh muscle cross-sectional area - Appendicular muscle mass - Physical performance

\begin{abstract}
Background/Aims: Our study aims to evaluate the association between thigh muscle crosssectional area (TMA) using computed tomography (CT), or appendicular skeletal muscle mass (ASM) using dual energy X-ray absorptiometry (DEXA), and physical performance levels in hemodialysis (HD) patients. Methods: Patients were included if they were on HD for $\geq 6$ months $(n=84)$. ASM and TMA were adjusted to body weight $(B W, \mathrm{~kg})$ or height ${ }^{2}\left(\mathrm{Ht}^{2}, \mathrm{~m}^{2}\right)$. Each participant performed a short physical performance battery test (SPPB), a sit-to-stand for 30 second test (STS30), a 6-minute walk test (6-MWT), a timed up and go test (TUG), and hand grip strength (HGS) test. Results: Correlation coefficients for SPPB, GS, 5STS, STS30, 6-MWT, and TUG were highest in TMA/BW. Results from partial correlation or linear regression analyses displayed similar trends to those derived from Pearson's correlation analyses. An increase in TMA/BW or TMA/ $\mathrm{Ht}^{2}$ was associated with a decreased odds ratio of low SPPB, GS, or HGS in multivariate analyses. Indices using DEXA were associated with a decreased odds ratio of a low HGS only in multivariate analysis. Conclusion: TMA indices using CT may be more valuable in predicting physical performance or strength in HD patients.
\end{abstract}

\section{Introduction}

Chronic kidney disease, caused by various factors such as diabetes mellitus (DM), hypertension, glomerulonephritis, or age, is a well-known public health problem. Chronic 


\section{Kidney \\ Blood Pressure Research}

kidney disease can progress to end-stage renal disease, which then requires renal replacement therapy. Hemodialysis (HD) is the most commonly used modality among renal replacement therapies $[1,2]$. HD patients are exposed to various factors including uremic toxins, metabolic disturbances, and inflammatory agents [3]. These are associated with early ageing processes in HD patients. Therefore, HD patients have a rapid decline in muscle mass leading to low physical performance as compared to a healthy population. Previous studies show that the prevalence of sarcopenia ranges from approximately $3.6 \%$ to $31.5 \%$ in HD patients [4-6]. Sarcopenia is associated with an adverse outcome; therefore, monitoring of muscle mass through accurate measurements, is important to predict low physical performance.

Decline in muscle mass is associated with low muscle strength, which leads to poor physical performance. However, two unresolved issues remain regarding the association between muscle mass and levels of physical performance. First, previous studies have conflicting results regarding the association between muscle mass measurements and physical performance [7-12]. Second, there is no consensus regarding the optimal methods for predicting muscle mass in HD patients. Dual X-ray absorptiometry (DEXA) is the most commonly used measurement for predicting muscle mass, but HD patients have a volume overload status and insulin resistance associated with fat mass accumulation. These factors may be associated with a more limited capacity to accurately predict muscle mass in HD patients $[13,14]$. Thigh muscle cross-sectional area (TMA) using computed tomography (CT) is a well-known, accurate method for predicting muscle mass. However, there are few studies regarding the comparison between TMA using CT and physical performance in HD patients. Our study aims address these issues and to evaluate the association between TMA using CT, or appendicular skeletal muscle mass (ASM) using DEXA, and physical performance levels in HD patients.

\section{Materials and Methods}

\section{Study population}

This study was conducted in CHA Gumi Medical Center, Republic of Korea, from 1 15t January 2015 to $30^{\text {th }}$ December 2015. Patients were included if they were over 20 years-old, on dialysis for $\geq 6$ months, with no history of hospitalization in the previous 3 months, able to ambulate without an assistive device, able to communicate with the interviewer, and able to provide informed consent. A total of 84 patients were enrolled. None of the participants were taking opioids, antihistamines, or antidepressants, which can be associated with decreased physical activity and cognitive function. This study was approved by the Institutional Review Board of CHA Gumi Medical Center (IRB number: 12-07) and all participants provided written informed consent.

\section{Study variables}

Demographic and laboratory data collected at enrollment included the following: age, sex, hemoglobin level (g/dL), Kt/Vurea, high-sensitivity C-reactive protein (hs-CRP, mg/dL), serum albumin level (g/dL), dialysis vintage, and the presence of DM. Kt/Vurea was calculated using following formula: Kt/Vurea $=-\mathrm{Ln}$ $(\mathrm{R}-0.008 \times \mathrm{t})+(4-3.5 \times \mathrm{R}) \times \mathrm{UF} / \mathrm{W}$ (R: post-dialysis blood urea nitrogen/pre-dialysis blood urea nitrogen, t: dialysis duration in hours, UF: ultrafiltration volume in liters, W: post-dialysis body weight in kilograms) [15]. If a patient had a self-reported history and a medical record of DM diagnosis or medication, the patient was defined as having DM.

\section{Assessment of muscle mass}

We performed two muscle mass measurements using DEXA and CT. Two measurements were performed after the midweek HD session. DEXA was measured using a Prodigy Advance instrument and performed by a technologist while each participant was in a supine position and clothed in a light gown (GE Medical Systems Lunar, Madison, WI, USA). ASM (kg) was defined as the sum of both upper extremities and lower extremities. After the DEXA measurement, each participant underwent a multi-slice, mid-thigh 


\section{Kidney Blood Pressure Research}

Kang et al.: Physical Performance in Dialysis Patients

CT scan using a 320-slice CT scanner (Aquilion ONE; Toshiba Medical Systems Corp., Tokyo, Japan). An axial image was obtained at the midpoint of a line extending from the superior border of the patella to the greater trochanter ( $3 \mathrm{~mm}$ thickness, 5 slices). The images were analyzed using image analysis software (ImageJ 1.45S, National Institutes of Health, Bethesda, MD, USA). ASM and TMA were adjusted to body weight (BW, $\mathrm{kg}$ ) or height ${ }^{2}\left(\mathrm{Ht}^{2}, \mathrm{~m}^{2}\right)$.

\section{Assessment of physical performance}

In our study, each participant performed a short physical performance battery test (SPPB), a sit-tostand for 30 second test (STS30), a 6-minute walk test (6-MWT), a timed up and go test (TUG), and hand grip strength (HGS) test. The SPPB test, which consisted of assessing the usual gait speed (GS), a sit-to-stand test performed 5 times (5STS), and balance tests, were calculated using previously defined methods, and scores ranged from 0 to 12 [16]. For GS, the participants were asked to walk a 4-meter distance and the time taken (in seconds) to walk the distance was recorded. For the 5STS test, each participant was seated in a chair with arms crossed and hands touching the shoulders [17]. The participants were asked to stand up and sit down 5 times as quickly as possible, and the time taken in seconds was recorded. For the STS30 test, the participants were seated in a chair with their arms crossed and hands touching the shoulders. Scores were defined as the number of stands a person could complete in 30 seconds without using their arms to stand [18]. For the 6-MWT, participants were asked to walk at their best pace for 6 minutes, and the distance they covered was recorded in meters [19]. For the TUG test, the participants were instructed to rise from an arm-chair, walk 3 meters, turn around, return, and sit down [20]. The time in seconds was recorded. For the HGS test, 3 trials were performed on the dominant hand using a manual hydraulic dynamometer (Jamar Hydraulic hand dynamometer; Sammons Preston, Chicago, USA). Over the 3 trials, maximum strength was recorded. We also defined a low physical performance group for the SPPB, GS, and HGS tests. The low SPPB group was defined as scoring $\leq 10$. The low GS group was defined as scoring $\leq 1 \mathrm{~m} / \mathrm{s}$ [21]. The low HGS group was defined as scoring $<26 \mathrm{~kg}$ for men and $<18 \mathrm{~kg}$ for women [22].

\section{Statistical analyses}

The data were analyzed using the statistical software SPSS version 21 (Chicago, IL, USA). Categorical variables were expressed as both counts and percentages. Continuous variables were expressed as mean \pm standard deviation (SD) or mean \pm standard error (SE). For continuous variables, means were compared using the t-test. Multivariate analysis was performed using analysis of covariance. Correlation between 2 continuous variables was assessed using Pearson's or partial correlation analyses. Linear regression analysis was performed to assess the independent predictors of each physical performance. Logistic regression analyses were used to estimate the odds ratios and 95\% confidence intervals (CI), which were used to determine the relationship between low physical performance groups and muscle mass indices. Multivariate analysis was adjusted for age, sex, and DM. The level of statistical significance was set at $P<$ 0.05 .

\section{Results}

\section{Participants' clinical characteristics}

The mean participant age was $55.6 \pm 12.8$ years (Table 1). The proportion of males was $52.4 \%$. The mean value of ASM, ASM/BW, and $\mathrm{ASM} / \mathrm{Ht}^{2}$ was $17.4 \pm 3.8 \mathrm{~kg}, 28.1 \pm 4.5 \%, 6.58$ $\pm 0.97 \mathrm{~kg} / \mathrm{m}^{2}$, respectively. The mean value of TMA, TMA/BW, and TMA/ $\mathrm{Ht}^{2}$ was $97.2 \pm 23.2$ $\mathrm{cm}^{2}, 1.56 \pm 0.25 \mathrm{~cm}^{2} / \mathrm{kg}$, and $36.9 \pm 7.04 \mathrm{~cm}^{2} /$ $\mathrm{m}^{2}$, respectively. The proportions of participant with low SPPB, GS, and HGS was 25 (29.8\%), 55 (65.5\%), and 15 (17.9\%), respectively.
Table 1. Participants' clinical characteristics

\begin{tabular}{lc}
\hline & $(\mathrm{n}=84)$ \\
\hline Age (years) & $55.6 \pm 12.8$ \\
Sex (male, \%) & $44(52.4 \%)$ \\
Diabetes mellitus (\%) & $44(52.4 \%)$ \\
Dialysis vintage (years) & $4.6 \pm 5.1$ \\
Hemoglobin (mg/dL) & $11.0 \pm 0.6$ \\
Serum albumin (g/dL) & $3.8 \pm 0.3$ \\
High sensitivity C-reactive protein (mg/dL) & $0.42 \pm 0.60$ \\
Kt/Vurea & $1.36 \pm 0.31$ \\
\hline
\end{tabular}




\section{Kidney Bloód Pressure Research}

Association between various muscle mass indices and physical performance

Pearson's correlation coefficients for SPPB, GS, 5STS, STS30, 6-MWT, and TUG were highest in TMA/BW (Table 2). The correlation coefficient values for SPPB, GS, STS5, STS30, 6-MWT, and TUG were 0.397, 0.559, $-0.326,0.497,0.531$, and -0.472 , respectively. The correlation coefficient for HGS was highest in TMA $(r=0.614)$. Results from partial correlation or linear regression analyses displayed similar trends to those derived from Pearson's correlation analyses (Table 3).

An increase in TMA/BW was associated with a decreased odds ratio of low SPPB, GS, or HGS in both univariate and multivariate analyses (Table 4). An increase in TMA/ $\mathrm{Ht}^{2}$ was associated with a decreased odds ratio of low SPPB, GS, or HGS in multivariate analysis. Indices using DEXA were associated with a decreased odds ratio of a low HGS only in multivariate analysis.

Comparison of indices between participants with normal and poor physical performance

For the SPPB test, univariate and multivariate analyses showed that there was no significant difference in indices using DEXA between participants with normal and low SPPB scores (Table 5). TMA/BW and TMA/ $\mathrm{Ht}^{2}$ were greater in participants with a normal SPPB score than in those with a low SPPB. For the GS test, univariate analysis showed that all indices were greater in participants with a normal GS than in those with a low GS. However, for multivariate analysis, statistical significances were observed only in $\mathrm{ASM} / \mathrm{Ht}^{2}$ and indices

Table 2. Correlation between various muscle mass indices and physical performance. Data were expressed as correlation coefficients. Partial correlations were adjusted for age, sex, and diabetes mellitus. ${ }^{*} \mathrm{P}<0.05$. Abbreviations: ASM, appendicular skeletal muscle mass; ASM/BW, appendicular skeletal muscle mass per body weight; ASM $/ \mathrm{Ht}^{2}$, appendicular skeletal muscle mass per height squared; TMA, thigh muscle area; TMA/BW, thigh muscle area per body weight; TMA/Ht ${ }^{2}$, thigh muscle area per height squared; SPPB, short physical performance battery; GS, gait speed; 5STS, 5 times sit to stand test; STS30, sit to stand for 30 seconds test; 6-MWT, 6 minute walk test; TUG, timed up and go test; HGS, hand grip strength

\begin{tabular}{lcccccc}
\hline & ASM & ASM/BW & ASM/Ht & TMA & TMA/BW & TMA/Ht $^{2}$ \\
\hline $\begin{array}{l}\text { Pearson } \\
\text { correlation }\end{array}$ & & & & & & \\
SPPB & 0.074 & 0.191 & 0.112 & 0.203 & $0.397^{*}$ & $0.259^{*}$ \\
GS & $0.380^{*}$ & $0.300^{*}$ & $0.355^{*}$ & $0.517^{*}$ & $0.559^{*}$ & $0.485^{*}$ \\
5STS & -0.088 & -0.172 & -0.150 & -0.197 & $-0.326^{*}$ & $-0.260^{*}$ \\
STS30 & 0.177 & 0.173 & $0.237^{*}$ & $0.393^{*}$ & $0.497^{*}$ & $0.476^{*}$ \\
6-MWT & $0.268^{*}$ & $0.359^{*}$ & $0.246^{*}$ & $0.359^{*}$ & $0.531^{*}$ & $0.330^{*}$ \\
TUG & -0.177 & $-0.243^{*}$ & -0.179 & $-0.324^{*}$ & $-0.472^{*}$ & $-0.333^{*}$ \\
HGS & $0.574^{*}$ & $0.412^{*}$ & $0.502^{*}$ & $0.614^{*}$ & $0.543^{*}$ & $0.492^{*}$ \\
Partial & & & & & & \\
correlation & & & & & & \\
SPPB & 0.035 & 0.144 & 0.166 & 0.173 & $0.347^{*}$ & $0.235^{*}$ \\
GS & 0.220 & 0.199 & 0.220 & $0.406^{*}$ & $0.453^{*}$ & $0.398^{*}$ \\
5STS & -0.090 & -0.171 & -0.177 & -0.199 & $-0.319^{*}$ & $-0.252^{*}$ \\
STS30 & 0.007 & 0.037 & 0.147 & $0.317^{*}$ & $0.437^{*}$ & $0.430^{*}$ \\
6-MWT & 0.117 & $0.257^{*}$ & 0.133 & $0.233^{*}$ & $0.454^{*}$ & $0.235^{*}$ \\
TUG & -0.050 & -0.145 & -0.097 & $-0.241^{*}$ & $-0.409^{*}$ & $-0.270^{*}$ \\
HGS & $0.255^{*}$ & 0.070 & $0.235^{*}$ & $0.386^{*}$ & $0.326^{*}$ & $0.349^{*}$ \\
\hline
\end{tabular}




\section{Kidney Blood Pressure Research}

Kang et al.: Physical Performance in Dialysis Patients

Table 3. Linear regression analyses of physical performances based on various muscle mass indices. Data were expressed as standardized $\beta \pm$ standard errors. The multivariate analyses were adjusted for age, sex, and diabetes mellitus. ${ }^{*} \mathrm{P}<0$ 0.05.Abbreviations: ASM, appendicular skeletal muscle mass; ASM/BW, appendicular skeletal muscle mass per body weight; $\mathrm{ASM} / \mathrm{Ht}^{2}$, appendicular skeletal muscle mass per height squared; TMA, thigh muscle area; TMA/BW, thigh muscle area per body weight; $\mathrm{TMA} / \mathrm{Ht}^{2}$, thigh muscle area per height squared; SPPB, short physical performance battery; GS, gait speed; 5STS, 5 times sit to stand test; STS30, sit to stand for 30 seconds test; 6-MWT, 6 minute walk test; TUG, timed up and go test; HGS, hand grip strength

\begin{tabular}{lcccccc}
\hline & ASM & ASM/BW & ASM/Ht & TMA & TMA/BW & TMA/Ht \\
\hline Univariate & & & & & & \\
SPPB & $0.074 \pm 0.047$ & $0.191 \pm 0.039$ & $0.112 \pm 0.184$ & $0.203 \pm 0.008^{*}$ & $0.397 \pm 0.649^{*}$ & $0.259 \pm 0.025^{*}$ \\
GS & $0.380 \pm 0.005^{*}$ & $0.300 \pm 0.005^{*}$ & $0.355 \pm 0.021^{*}$ & $0.517 \pm 0.001^{*}$ & $0.559 \pm 0.071^{*}$ & $0.485 \pm 0.003^{*}$ \\
5STS & $-0.088 \pm 0.179$ & $-0.172 \pm 0.148$ & $-0.150 \pm 0.692$ & $-0.197 \pm 0.029$ & $-0.326 \pm 2.532^{*}$ & $-0.260 \pm 0.093^{*}$ \\
STS30 & $0.177 \pm 0.164$ & $0.173 \pm 0.138$ & $0.237 \pm 0.631^{*}$ & $0.393 \pm 0.025^{*}$ & $0.497 \pm 2.157^{*}$ & $0.476 \pm 0.079^{*}$ \\
6-MWT & $0.268 \pm 3.171^{*}$ & $0.359 \pm 2.580^{*}$ & $0.246 \pm 12.446^{*}$ & $0.359 \pm 0.502^{*}$ & $0.531 \pm 41.646^{*}$ & $0.330 \pm 1.673^{*}$ \\
TUG & $-0.177 \pm 0.058$ & $-0.243 \pm 0.048^{*}$ & $-0.179 \pm 0.227$ & $-0.324 \pm 0.009^{*}$ & $-0.472 \pm 0.777^{*}$ & $-0.333 \pm 0.030^{*}$ \\
HGS & $0.574 \pm 0.176^{*}$ & $0.412 \pm 0.164^{*}$ & $0.502 \pm 0.724^{*}$ & $0.614 \pm 0.028^{*}$ & $0.543 \pm 2.691^{*}$ & $0.492 \pm 0.101^{*}$ \\
Multivariate & & & & & & \\
SPPB & $0.068 \pm 0.067$ & $0.168 \pm 0.044$ & $0.143 \pm 0.219$ & $0.210 \pm 0.009$ & $0.373 \pm 0.717^{*}$ & $0.236 \pm 0.025^{*}$ \\
GS & $0.264 \pm 0.008$ & $0.114 \pm 0.005$ & $0.234 \pm 0.025$ & $0.443 \pm 0.001^{*}$ & $0.456 \pm 0.080^{*}$ & $0.378 \pm 0.003^{*}$ \\
5STS & $-0.155 \pm 0.265$ & $-0.208 \pm 0.175$ & $-0.228 \pm 0.860$ & $-0.254 \pm 0.036$ & $-0.359 \pm 2.878^{*}$ & $-0.265 \pm 0.100^{*}$ \\
STS30 & $0.045 \pm 0.238$ & $0.057 \pm 0.159$ & $0.187 \pm 0.772$ & $0.386 \pm 0.031^{*}$ & $0.472 \pm 2.442^{*}$ & $0.433 \pm 0.083^{*}$ \\
6-MWT & $0.170 \pm 4.354$ & $0.275 \pm 2.838^{*}$ & $0.156 \pm 14.279$ & $0.262 \pm 0.588^{*}$ & $0.453 \pm 44.707^{*}$ & $0.221 \pm 1.650^{*}$ \\
TUG & $-0.079 \pm 0.079$ & $-0.158 \pm 0.053$ & $-0.116 \pm 0.259$ & $-0.271 \pm 0.011^{*}$ & $-0.411 \pm 0.828^{*}$ & $-0.256 \pm 0.030^{*}$ \\
HGS & $0.308 \pm 0.244^{*}$ & $0.072 \pm 0.169$ & $0.239 \pm 0.805^{*}$ & $0.377 \pm 0.032^{*}$ & $0.284 \pm 2.733^{*}$ & $0.288 \pm 0.092^{*}$ \\
\hline
\end{tabular}

Table 4. Logistic regression analyses of decreased physical performances according to various muscle mass indices. Data were expressed as odds ratio (95\% confidence interval) and odds ratios were defined as increase in one unit of each indices. The multivariate analyses were adjusted for age, sex, and diabetes mellitus. Abbreviations: ASM, appendicular skeletal muscle mass; ASM/BW, appendicular skeletal muscle mass per body weight; ASM/Ht², appendicular skeletal muscle mass per height squared; TMA, thigh muscle area; TMA/BW, thigh muscle area per body weight; TMA/ $\mathrm{Ht}^{2}$, thigh muscle area per height squared; SPPB, short physical performance battery; GS, gait speed; HGS, hand grip strength

\begin{tabular}{lcccccc}
\hline & $\mathrm{ASM}$ & $\mathrm{ASM} / \mathrm{BW}$ & $\mathrm{ASM} / \mathrm{Ht} \mathrm{t}^{2}$ & $\mathrm{TMA}$ & $\mathrm{TMA} / \mathrm{BW}$ & $\mathrm{TMA} / \mathrm{Ht}^{2}$ \\
\hline Univariate & & & & & & \\
Low SPPB & $0.98(0.87-1.12)$ & $0.94(0.84-1.04)$ & $0.90(0.55-1.46)$ & $0.99(0.97-1.01)$ & $0.03(0.00-0.38)$ & $0.96(0.90-1.03)$ \\
& $\mathrm{P}=0.803$ & $\mathrm{P}=0.227$ & $\mathrm{P}=0.659$ & $\mathrm{P}=0.425$ & $\mathrm{P}=0.006$ & $\mathrm{P}=0.273$ \\
Low GS & $0.78(0.68-0.91)$ & $0.87(0.78-0.97)$ & $0.37(0.21-0.66)$ & $0.98(0.95-1.00)$ & $0.04(0.00-0.58)$ & $0.93(0.86-1.01)$ \\
& $\mathrm{P}=0.001$ & $\mathrm{P}=0.011$ & $\mathrm{P}=0.001$ & $\mathrm{P}=0.069$ & $\mathrm{P}=0.018$ & $\mathrm{P}=0.100$ \\
Low HGS & $0.78(0.64-0.94)$ & $0.86(0.74-0.99)$ & $0.37(0.18-0.75)$ & $0.98(0.96-1.00)$ & $0.02(0.00-0.22)$ & $0.92(0.85-0.99)$ \\
& $\mathrm{P}=0.011$ & $\mathrm{P}=0.045$ & $\mathrm{P}=0.006$ & $\mathrm{P}=0.097$ & $\mathrm{P}=0.002$ & $\mathrm{P}=0.036$ \\
Multivariate & & & & & & \\
Low SPPB & $1.00(0.82-1.21)$ & $0.94(0.82-1.09)$ & $0.86(0.44-1.65)$ & $0.98(0.95-1.01)$ & $0.01(0.00-0.26)$ & $0.91(0.83-0.99)$ \\
& $\mathrm{P}=0.988$ & $\mathrm{P}=0.403$ & $\mathrm{P}=0.642$ & $\mathrm{P}=0.111$ & $\mathrm{P}=0.005$ & $\mathrm{P}=0.045$ \\
Low GS & $0.87(0.71-1.07)$ & $0.95(0.84-1.09)$ & $0.51(0.25-1.03)$ & $0.96(0.93-0.99)$ & $0.04(0.00-0.45)$ & $0.89(0.81-0.97)$ \\
& $\mathrm{P}=0.193$ & $\mathrm{P}=0.483$ & $\mathrm{P}=0.062$ & $\mathrm{P}=0.015$ & $\mathrm{P}=0.010$ & $\mathrm{P}=0.007$ \\
Low HGS & $0.53(0.36-0.77)$ & $0.81(0.67-0.98)$ & $0.17(0.06-0.50)$ & $0.91(0.86-0.96)$ & $0.01(0.00-0.22)$ & $0.82(0.72-0.94)$ \\
& $\mathrm{P}=0.001$ & $\mathrm{P}=0.030$ & $\mathrm{P}=0.001$ & $\mathrm{P}=0.001$ & $\mathrm{P}=0.005$ & $\mathrm{P}=0.003$ \\
\hline
\end{tabular}

using TMA. For the HGS test, there were significant differences in all indices between participants with normal and low HGS scores on both univariate and multivariate analyses.

\section{Discussion}

In univariate and multivariate analyses, our study showed a consistent superiority in predicting physical performance or strength indicators of TMA indices using CT, compared 


\section{Kidney Blood Pressure Research}

Kang et al.: Physical Performance in Dialysis Patients

Table 5. Changes in muscle mass indices according to physical performance status. Data were expressed as mean \pm standard deviations for univariate analysis and mean \pm standard errors for multivariate analysis. Univariate analysis and multivariate analysis were performed using t-test and analysis of covariance, respectively. Multivariate analyses were adjusted for age, sex, and diabetes mellitus. Abbreviations: ASM, appendicular skeletal muscle mass; ASM/BW, appendicular skeletal muscle mass per body weight; ASM/ $\mathrm{Ht}^{2}$, appendicular skeletal muscle mass per height squared; TMA, thigh muscle area; TMA/BW, thigh muscle area per body weight; TMA/ $\mathrm{Ht}^{2}$, thigh muscle area per height squared; SPPB, short physical performance battery; GS, gait speed; HGS, hand grip strength

\begin{tabular}{|c|c|c|c|c|c|c|c|c|c|}
\hline & \multicolumn{3}{|c|}{ SPPB } & \multicolumn{3}{|c|}{ GS } & \multicolumn{3}{|c|}{ HGS } \\
\hline & Low & Normal & $\mathrm{P}$-value & Low & Normal & P-value & Low & Normal & P-value \\
\hline \multicolumn{10}{|l|}{ Univariate } \\
\hline ASM & $17.3 \pm 4.2$ & $17.5 \pm 3.7$ & 0.806 & $16.3 \pm 3.1$ & $19.4 \pm 4.2$ & $<0.001$ & $15.1 \pm 3.0$ & $17.9 \pm 3.8$ & 0.008 \\
\hline ASM/BW & $27.2 \pm 4.4$ & $28.5 \pm 4.5$ & 0.228 & $27.1 \pm 4.2$ & $29.9 \pm 4.7$ & 0.008 & $25.9 \pm 3.7$ & $28.6 \pm 4.6$ & 0.039 \\
\hline $\mathrm{ASM} / \mathrm{Ht}^{2}$ & $6.51 \pm 0.99$ & $6.61 \pm 0.97$ & 0.663 & $6.30 \pm 0.82$ & $7.11 \pm 1.03$ & $<0.001$ & $5.93 \pm 0.78$ & $6.72 \pm 0.96$ & 0.004 \\
\hline TMA & $90.7 \pm 24.3$ & $100.0 \pm 22.4$ & 0.094 & $89.4 \pm 18.6$ & $112.0 \pm 24.1$ & $<0.001$ & $80.4 \pm 12.5$ & $100.9 \pm 23.4$ & 0.002 \\
\hline TMA/BW & $1.42 \pm 0.19$ & $1.62 \pm 0.25$ & 0.001 & $1.48 \pm 0.22$ & $1.72 \pm 0.24$ & $<0.001$ & $1.39 \pm 0.15$ & $1.60 \pm 0.26$ & 0.002 \\
\hline TMA/Ht ${ }^{2}$ & $34.3 \pm 6.5$ & $37.9 \pm 7.0$ & 0.031 & $34.6 \pm 6.2$ & $41.2 \pm 6.7$ & $<0.001$ & $31.9 \pm 4.7$ & $38.0 \pm 7.0$ & 0.002 \\
\hline \multicolumn{10}{|c|}{ Multivariate } \\
\hline ASM & $17.4 \pm 0.5$ & $17.4 \pm 0.3$ & 0.967 & $17.1 \pm 0.4$ & $18.0 \pm 0.5$ & 0.150 & $15.1 \pm 0.6$ & $17.9 \pm 0.3$ & $<0.001$ \\
\hline ASM/BW & $27.5 \pm 0.8$ & $28.3 \pm 0.5$ & 0.421 & $27.9 \pm 0.5$ & $28.5 \pm 0.8$ & 0.478 & $26.2 \pm 1.0$ & $28.5 \pm 0.4$ & 0.032 \\
\hline $\mathrm{ASM} / \mathrm{Ht}^{2}$ & $6.52 \pm 0.16$ & $6.61 \pm 0.10$ & 0.637 & $6.45 \pm 0.11$ & $6.83 \pm 0.15$ & 0.046 & $5.93 \pm 0.19$ & $6.72 \pm 0.09$ & $<0.001$ \\
\hline TMA & $91.9 \pm 3.8$ & $99.5 \pm 2.4$ & 0.105 & $92.9 \pm 2.5$ & $105.3 \pm 3.5$ & 0.007 & $81.5 \pm 4.4$ & $100.6 \pm 2.0$ & $<0.001$ \\
\hline TMA/BW & $1.45 \pm 0.04$ & $1.61 \pm 0.03$ & 0.003 & $1.51 \pm 0.03$ & $1.67 \pm 0.04$ & 0.004 & $1.41 \pm 0.06$ & $1.60 \pm 0.03$ & 0.003 \\
\hline $\mathrm{TMA} / \mathrm{Ht}^{2}$ & $34.5 \pm 1.3$ & $37.9 \pm 0.9$ & 0.045 & $35.2 \pm 0.9$ & $40.1 \pm 1.2$ & 0.002 & $32.2 \pm 1.6$ & $37.9 \pm 0.8$ & 0.002 \\
\hline
\end{tabular}

with ASM indices using DEXA, in HD patients. These results suggest that TMA indices using CT would be valuable for predicting physical performance in HD patients.

HD patients have higher insulin resistance and prevalence of DM than the general population, resulting in changes in body composition [23-25]. In HD patients, various factors including inflammation lead to insulin resistance, which is associated with increased catabolism and decreased anabolism in the muscles [26,27]. These lead to sarcopenia or protein-energy wasting. Previous studies showed that prevalence of protein-energy wasting was higher in HD patients with insulin resistance or DM than in those without insulin resistance or DM [26-28]. Fat mass is associated with development of insulin resistance or DM. However, the association between insulin resistance and change in fat mass is complex. Patients with insulin resistance or DM have decreased lipolysis and increased adipogenesis, but HD patients are prone to low energy intake and increased catabolism [26, 27, 29]. Although HD patients with adequate or high energy intake have increased fat mass, most HD patients have decreased or stable fat mass when on dialysis [30-32].

End-stage renal disease is associated with a rapid decline in muscle mass, which leads to high mortality and low quality of life $[3,4,33,34]$. Therefore, early detection of decline in muscle mass and accurate measurement of muscle mass is important to improve the prognosis of dialysis patients. In non-dialysis patients, muscle mass is measured using various methods such as DEXA, bioimpedance analysis, CT, or creatinine-based equations. DEXA is a useful method for predicting muscle mass in the general population. However, compared with non-dialysis patients, HD patients are prone to overhydration [35]. Overhydration in HD patients can lead to overestimation of lean mass. Formica et al. investigated fat-free mass before and after ultrafiltration in HD patients. That study showed that gravimetric weight loss almost always consists of a decrease in lean mass [36]. Although measurements using DEXA are performed after an HD session, inappropriate dry weight is associated with inaccurate measurement of muscle mass. In addition, because lean mass measurement using DEXA is calculated after subtracting bone mass and fat mass from total mass, lean mass from DEXA includes skin and fat-free components of adipose tissue [37]. Levine et al. showed an approximately $6 \%$ to $13 \%$ overestimate for muscle mass using CT. This is associated with an inherent overestimation of muscle mass calculation using DEXA [37]. 


\section{Kidney Blood Pressure Research}

Although there are few studies regarding the comparison between DEXA and CT in the measurement of muscle mass according to volume overload, CT would be less likely overestimate muscle mass estimation compared with DEXA due to the division of total volume overload into subcutaneous tissue and intramuscular tissue. Kalima et al. investigated volume distribution in chronic venous insufficiency patients, clinically similar to overhydrated HD patients [38]. They showed that volume overload of HD patients was distributed into the subcutaneous tissue in $25 \%$ and into the intramuscular tissue in $65 \%$. These results reveal that measurement using CT would reduce overestimation of muscle mass by volume overload compared with using DEXA. In addition, TMA using CT can exclude skin mass.

There are conflicting results regarding the association between muscle mass and physical performance. Although some studies have shown that the association between muscle mass and physical performance has a linear association, other studies have demonstrated a weak association between the two variables [8-11,39]. These heterogeneous results indicate the need of studies to use specified population groups, for example various diseased populations or ethnicities. To the best of our knowledge, the present study is the first study that compares muscle mass measurements using CT and DEXA for predicting physical performance in HD patients. We also considered body size and analyzed data adjusted for body weight or height squared. Our study shows that muscle strength using HGS is associated with both TMA indices using CT and with ASM indices using DEXA. TMA measured the lower extremity only, but a decrease of muscle mass in pathologic conditions such as in HD patients is likely to develop in both upper and lower extremities at the same time. Therefore, muscle mass in the lower extremity would correlate highly with muscle mass or strength in the upper extremity in HD patients. In addition, our results reveal a higher association of TMA indices using CT with HGS than ASM indices using DEXA. TMA indices using CT were superior to ASM indices using DEXA for predicting physical performance. Better estimation of muscle mass using CT may be associated with superiority in the prediction of HGS or physical performance. Adjusted data was a better predictor of physical performance than raw muscle mass, but there was no difference in prediction of physical performance among adjusted variables.

The limitations of the present study should be considered. Our study was performed in a single center and included a small number of patients. We did not calculate sample size to confirm statistical significance. We included all participants who provide informed consent. In addition, our study did not include data regarding volume status and muscle mass using standardized reference methods (e.g., total body potassium or nitrogen). A large prospective study, including additional parameters such as volume status and reference measurement methods for muscle mass, is warranted to overcome these limitations.

In conclusion, the present study demonstrates that TMA indices using CT may be more valuable in predicting physical performance or strength in HD patients. Our results can provide information regarding optimal muscle mass measurements for predicting physical performance or strength, which may be more closely associated with improved quality of life or prognosis.

\section{Acknowledgements}

This work wassupported by the Medical Research Center Program(2015R1A5A2009124) through the National Research Foundation of Korea (NRF) funded by the Ministry of Science, ICT and Future Planning.

\section{Disclosure Statement}

The authors declare that they have no Disclosure Statement. 


\section{Kidney \\ Blood Pressure Research}

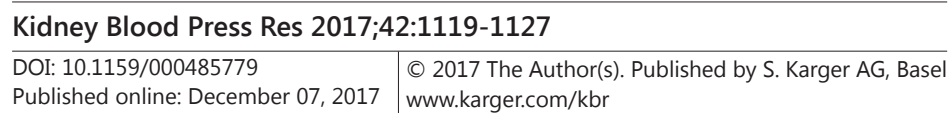

Kang et al.: Physical Performance in Dialysis Patients

\section{References}

1 US Renal Data System, USRDS 2015 Annual Data Report: Atlas of Chronic Kidney Disease in the United States, National Institutes of Health, National Institute of Diabetes and Digestive and Kidney Diseases, Bethesda, MD, 2015.

2 Jin DC: Current Renal Replacement Therapy in Korea. Proceedings of the $35^{\text {th }}$ Annual Spring Meeting of the Korean Society of Nephrology. 2015;Suppl1:117-136.

3 Fouque D, Kalantar-Zadeh K, Kopple J, Cano N, Chauveau P, Cuppari L, Franch H, Guarnieri G, Ikizler TA, Kaysen G, Lindholm B, Massy Z, Mitch W, Pineda E, Stenvinkel P, Treviño-Becerra A, Wanner C: A proposed nomenclature and diagnostic criteria for protein-energy wasting in acute and chronic kidney disease. Kidney Int 2008;73:391-398.

4 Kim JK, Choi SR, Choi MJ, Kim SG, Lee YK, Noh JW, Kim HJ, Song YR: Prevalence of and factors associated with sarcopenia in elderly patients with end-stage renal disease. Clin Nutr 2014;33:64-68.

5 Bataille S, Serveaux M, Carreno E, Pedinielli N, Darmon P, Robert A: The diagnosis of sarcopenia is mainly driven by muscle mass in hemodialysis patients. Clin Nutr 2017;36:1654-1660.

6 Kittiskulnam P, Carrero JJ, Chertow GM, Kaysen GA, Delgado C, Johansen KL: Sarcopenia among patients receiving hemodialysis: weighing the evidence. J Cachexia Sarcopenia Muscle 2017;8:57-68.

7 Lauretani F, Russo CR, Bandinelli S, Bartali B, Cavazzini C, Di Iorio A, Corsi AM, Rantanen T, Guralnik JM, Ferrucci L: Age-associated changes in skeletal muscles and their effect on mobility: an operational diagnosis of sarcopenia. J Appl Physiol 2003;95:1851-1860.

-8 Rolland Y, Czerwinski S, Abellan Van Kan G, Morley JE, Cesari M, Onder G, Woo J, Baumgartner R, Pillard F, Boirie Y, Chumlea WM, Vellas B: Sarcopenia: its assessment, etiology, pathogenesis, consequences and future perspectives. J Nutr Health Aging 2008;12:433-450.

-9 Janssen I, Heymsfield SB, Ross R: Low relative skeletal muscle mass (sarcopenia) in older persons is associated with functional impairment and physical disability. J Am Geriatr Soc 2002;50:889-896.

-10 Reid KF, Naumova EN, Carabello RJ, Phillips EM, Fielding RA: Lower extremity muscle mass predicts functional performance in mobility-limited elders. J Nutr Health Aging 2008;12:493-498.

11 Visser M, Newman AB, Nevitt MC, Kritchevsky SB, Stamm EB, Goodpaster BH, Harris TB: Reexamining the sarcopenia hypothesis. Muscle mass versus muscle strength. Health, Aging, and Body Composition Study Research Group. Ann N Y Acad Sci 2000;904:456-461.

12 Kim KE, Jang SN, Lim S, Park YJ, Paik NJ, Kim KW, Jang HC, Lim JY: Relationship between muscle mass and physical performance: is it the same in older adults with weak muscle strength? Age Ageing 2012;41:799803.

13 Proctor DN, O’Brien PC, Atkinson EJ, Nair KS: Comparison of techniques to estimate total body skeletal muscle mass in people of different age groups. Am J Physiol 1999;277:E489-495.

14 Kang SH, Cho KH, Park JW, Yoon KW, Do JY: Comparison of bioimpedance analysis and dual-energy X-ray absorptiometry body composition measurements in peritoneal dialysis patients according to edema. Clin Nephrol 2013;79:261-268.

15 Daugirdas JT: Second generation logarithmic estimates of single-pool variable volume Kt/V: an analysis of error. J Am Soc Nephrol 1993;4:1205-1213.

-16 Guralnik JM, Simonsick EM, Ferrucci L, Glynn RJ, Berkman LF, Blazer DG, Scherr PA, Wallace RB: A short physical performance battery assessing lower extremity function: Association with self-reported disability and prediction of mortality and nursing home admission. J Gerontol 1994;49:M85-M94.

17 Lord SR, Murray SM, Chapman K, Munro B, Tiedemann A: Sit-to-stand performance depends on sensation, speed, balance, and psychological status in addition to strength in older people. J Gerontol A Biol Sci Med Sci 2002;57:M539-M543.

18 Macfarlane DJ, Chou KL, Cheng YH, Chi I: Validity and normative data for thirty second chair stand test in elderly community-dwelling Hong Kong Chinese. Am J Hum Biol 2006;18:418-421.

19 Guyatt GH, Sullivan MJ, Thompson PJ, Fallen EL, Puqsley SO, Taylor DW, Berman LB: The 6-minute walk: a new measure of exercise capacity in patients with chronic heart failure. Can Med Assoc J 1985;132:919923. 


\section{Kidney \\ Blood Pressure Research}

Kang et al.: Physical Performance in Dialysis Patients

20 Podsiadlo D, Richardson S: The timed "Up \& Go": a test of basic functional mobility for frail elderly persons. J Am Geriatr Soc 1991;39:142-148.

-21 Liu LK, Lee WJ, Liu CL, Chen LY, Lin MH, Peng LN, Chen LK: Age-related skeletal muscle mass loss and physical performance in Taiwan: implications to diagnostic strategy of sarcopenia in Asia. Geriatr Gerontol Int 2013;13:964-971.

22 Chen LK, Liu LK, Woo J, Assantachai P, Auyeung TW, Bahyah KS, Chou MY, Chen LY, Hsu PS, Krairit O, Lee JS, Lee WJ, Lee Y, Liang CK, Limpawattana P, Lin CS, Peng LN, Satake S, Suzuki T, Won CW, et al: Sarcopenia in Asia: consensus report of the Asian Working Group for Sarcopenia. J Am Med Dir Assoc 2014;15:95-101.

-23 DeFronzo RA, Alvestrand A, Smith D, Hendler R, Hendler E, Wahren J: Insulin resistance in uremia. J Clin Invest 1981;67:563-568.

-24 He WY, Wu F, Pang XX, Chen GJ, A LT, He L, Wang S, Tang CS, Zhang AH: Irisin is Associated with Urotensin II and Protein Energy Wasting in Hemodialysis Patients. Kidney Blood Press Res 2016;41:78-85.

25 Dungey M, Bishop NC, Young HM, Burton JO, Smith AC: The Impact of Exercising During Haemodialysis on Blood Pressure, Markers of Cardiac Injury and Systemic Inflammation--Preliminary Results of a Pilot Study. Kidney Blood Press Res 2015;40:593-604.

-26 de Costa JA, Ikizler TA: Inflammation and insulin resistance as novel mechanisms of wasting in chronic dialysis patients. Semin Dial 2009;22:652-657.

27 Noori N, Kopple JD: Effect of diabetes mellitus on protein-energy wasting and protein wasting in end-stage renal disease. Semin Dial 2010;23:178-184.

28 Bernardo AP, Oliveira JC, Santos O, Carvalho MJ, Cabrita A, Rodrigues A: Insulin Resistance in Nondiabetic Peritoneal Dialysis Patients: Associations with Body Composition, Peritoneal Transport, and Peritoneal Glucose Absorption. Clin J Am Soc Nephrol 2015;10:2205-2212.

29 Gastaldelli A: Role of beta-cell dysfunction, ectopic fat accumulation and insulin resistance in the pathogenesis of type 2 diabetes mellitus. Diabetes Res Clin Pract 2011;93:S60-S65.

-30 Kalantar-Zadeh K, Kuwae N, Wu DY, Shantouf RS, Fougue D, Anker SD, Block G, Kopple JD: Associations of body fat and its changes over time with quality of life and prospective mortality in hemodialysis patients. Am J Clin Nutr 2006;83:202-210.

31 Johansen KL, Kaysen GA, Young BS, Hung AM, da Silva M, Chertow GM: Longitudinal study of nutritional status, body composition, and physical function in hemodialysis patients. Am J Clin Nutr 2003;77:842-846.

32 Hung SC, Tarng DC: Adiposity and insulin resistance in nondiabetic hemodialysis patients: effects of high energy supplementation. Am J Clin Nutr 2009;90:64-69.

-33 Dziubek W, Kowalska J, Kusztal M, Rogowski Ł, Gołębiowski T, Nikifur M, Szczepańska-Gieracha J, ZembrońŁacny A, Klinger M, Woźniewski M: The Level of Anxiety and Depression in Dialysis Patients Undertaking Regular Physical Exercise Training--a Preliminary Study. Kidney Blood Press Res 2016;41:86-98.

-34 Grzegorzewska AE, Izdebska A, Niepolski L, Warchoł W, Jagodziński PP: Self-Reported Physical Activity, Quality of Life, and Psychological Status in Relation to Plasma 25-Hydroxyvitamin D Concentration in Patients Treated with Hemodialysis. Kidney Blood Press Res 2016;41:886-900.

-35 Wabel P, Moissl U, Chamney P, Jirka T, Machek P, Ponce P, Taborsky P, Tetta C, Velasco N, Vlasak J, Zaluska W, Wizemann V: Towards improved cardiovascular management: the necessity of combining blood pressure and fluid overload. Nephrol Dial Transplant 2008;23:2965-2971.

-36 Formica C, Atkinson MG, Nyulasi I, McKay J, Heale W, Seeman E: Body composition following hemodialysis: studies using dual-energy X-ray absorptiometry and bioelectrical impedance analysis. Osteoporos Int 1993;3:192-197.

-37 Levine JA, Abboud L, Barry M, Reed JE, Sheedy PF, Jensen MD: Measuring leg muscle and fat mass in humans: comparison of CT and dual-energy X-ray absorptiometry. J Appl Physiol (1985) 2000;88:452-456.

38 Kalima TV, Tallroth K, Somer J: Computed Tomography in the differential diagnosis of unilateral lower extremity oedemas. In: Progress in Lymphology X. Proceedings of the Xth International Congress of Lymphology. University Adelaide Press, Prague 1985:116-118.

-39 Newman AB, Haggerty CL, Goodpaster B, Harris T, Kritchevsky S, Nevitt M, Miles TP, Visser M, Health Aging and Body Composition Research Group: Strength and muscle quality in a well-functioning cohort of older adults: the Health, Aging and Body Composition Study. J Am Geriatr Soc 2003;51:323-330. 